\title{
Functions and dysfunctions of neuroplastin65 in the nervous system
}

\begin{abstract}
The enormous number and complexity of connections in the nervous system depend on many families of adhesion proteins. Cell adhesion molecules are extensively distributed among cells in the nervous system and their functions are indispensable for the formation of intercellular connections both during development and in the adult. Neuroplastin65 (NP65), a member of immunoglobulin super family cell adhesion molecules, has been shown to be important for neuritogenesis, synaptogenesis and the regulation of synaptic plasticity. Recent advances suggest it may involve in the maintenance of excitatory/inhibitory balance and dysfunctions of NP65 could probably affect several cognitive and emotional processes. Here we present the functions of NP65 and provide potential evidences for dysfunctions of neruoplastin65 in neuropsychiatric disorders of the nervous system.
\end{abstract}

Keywords: neuroplastin65, np65, neuritogenesis, synaptogenesis, synaptic plasticity, excitatory/inhibitory balance, neuropsychiatric disorders
Volume 3 Issue 2 - 2017

\author{
Qionglan Yuan, Huanhuan Li \\ Department of Neurology, Tongii University School of Medicine, \\ China
}

Correspondence: Qionglan Yuan, Department of Neurology, Shanghai Tongji hospital, Tongji University School of Medicine, 200065, China, Email yqiongl@tongji.edu.cn

Received: August 0I, 2017| Published:September 05, 2017
Abbreviations: NP65, neuroplatin65; NP55, neuropaltin55; CAMs, cell adhesion molecules; IgSF, immunoglobulin super family; FGFR, fibroblast growth factor receptors; DCC, deleted in colorectal cancer; NCAM, neural cell adhesion molecule;

\section{Introduction}

Cell adhesion molecules (CAMs) have essential roles in forming functional networks in the nervous system. CAMs can be classified into several families including immunoglobulin super family (IgSF), cadherin family, integrins and selections. ${ }^{1}$ Neuroplastins are transmembrane glycoprotein's that belong to IgSF. Neuroplastin65 (NP65) and neuroplastin55 (NP55) are produced by NPTN gene by alternative splicing. ${ }^{2}$ NP55 is widely distributed in various tissues. However, NP65 is restrictively expressed in the nervous system.

In this review, we mainly focus on one of the neuroplastin isoforms-NP65. We provide an overview of the functions of NP65 in nervous system as well as the underlying cellular mechanisms. Finally, we discuss recent findings supporting altered functions of NP65 in several neuropsychiatric disorders and highlight some of the challenges in future researches.

\section{Structure of NP65}

NP65 is originally identified as gp65, a synaptic-membrane glycoprotein with molecular mass of $65 \mathrm{kDa} .{ }^{3}$ There are three Ig-like domains located in the N-terminal extracellular domain of NP65. The first Ig domain of NP65 belongs to C2 set, which contains approximately 76 amino acids and characterizes it from another neuroplastin isoform-NP55. Several glycosylation sites have been recognized in the ectodomain of NP65. The molecular mass of deglycosylated NP65 is approximately $40 \mathrm{kDa}$. NP65 has a 21 amino acids transmembrane domain downstream of the extracellular domain and followed by a 34 amino acids cytoplasmic domain. Genetic studies have detected a four amino acids (DDEP) insertion in the C-terminal intracellular domains of NP65. Thus, NP65 can be existed in two isoforms: NP65+DDEP and NP65 -DDEP, respectively. ${ }^{4}$
Protein blast research reveals that NP65 shares sequence similarities with several IgSF members. The highest similarities in sequence of NP65 are NP55 and basigin. NP65 also shares similarities with fibroblast growth factor receptors (FGFR) and other immunoglobulin cell adhesion molecules (Ig CAMs), including neural cell adhesion molecule L1 (L1), neural cell adhesion molecule (NCAM), contacting and netrin-1 receptor Deleted in Colorectal Cancer (DCC). By crystallography analysis, it has been shown that the secondary structure of NP65 contains several $\beta$-sheets and $\alpha$-helixes formed by each extracellular Ig domain. In the crystal structure of NP65, the second Ig set (Ig2) is oriented at an angle of $45^{\circ}$ to the $\mathrm{C} 2$ set and with the third $\operatorname{Ig}$ set (Ig3) oriented about the same angel to $\operatorname{Ig} 2 .^{5}$

\section{Localization of NP65}

In rodents, NP65 is expressed in the brain, retina and ear. NP65 is barely detectable in other tissues. Previous studies have confirmed that NP65 is enriched in cerebral cortex, hippocampus, striatum and amygdale. ${ }^{3}$ Among the cerebral cortex, NP65 is mainly located in layers of II, III and Vb/VI. In the hippocampus, NP65 is highly expressed in the CA1 region and DG region compared to other hippocampal regions such as CA3. ${ }^{6,7}$ There are only low levels of NP65 in the thalamus and hypothalamus. NP65 is not detected in cerebellum, pons and medulla and spinal cord. In rat retina, NP65 is distributed in both the inner and outer plexiform layer. ${ }^{4}$ NP65 is also presented in the cochlea of the mouse inner ear. ${ }^{8}$

In the human brain, NP65 is also highly expressed in cortex, hippocampus and putamen. In contrast, the strongest expression of NP65 is appeared in cerebellum and hypothalamus in human brain. NP65 expression does not show regional differences in cerebral cortex and hippocampus. ${ }^{9}$ It remains unclear whether these differences of NP65 expressions between the human and rodent brain influence its functions.

NP65 is predominately expressed in neurons in the rodent and human brain. ${ }^{3,9,10}$ In neurons, NP65 is a synaptic-enriched protein, and mostly concentrated in the postsynaptic densities. Neuoplatin65 
may also express in some presynaptic regions. Immuno-histochemical stains performed on human brain revealed that NP65 is visible in the membrane of cell bodies and dendrites as well as nerve fibers. NP65 is also reported to be expressed in glia of the rat optic nerve. ${ }^{4}$ However, NP65 is not expressed by glia in the human brain. ${ }^{9}$

\section{Functions of NP65}

NP65 is barely expressed during the embryonic development. ${ }^{5,11}$ In the rat forebrain, NP65 expression level increases slowly from postnatal day 1 to day 12. After then, NP65 level starts to increase dramatically and eventually reaches to the adult level at postnatal day 24. ${ }^{3}$ In the human brain, NP65 expression levels start to increase at later stages of development until adulthood and decline to a consistent level thereafter. ${ }^{11}$ Interestingly, NP65 expression level in the hippocampus at postnatal day is consistent with that of two synaptic proteins: synaptophysin and PSD-95, respectively. ${ }^{12}$ Evidences provided by developmental profile of NP65 suggest its possible roles in neuronal development. Indeed, there is accumulating findings indicate that NP65 is required for neurite outgrowth and the formation of synapse.

NP65 and nerite outgrowth: Recent studies have been revealed the roles of NP65 in neurite outgrowth. Homophilic bindings between NP65 may be involved in neurite growth during neuronal maturation (Figure 1A). Recombinant proteins derived from the ectodomain of NP65 (ectoNP65) and the first Ig domain (enplastin) are shown to be able to induce neurite outgrowth in cultured rat hippocampus neurons and cerebellum granule cells. Mechanism underlying NP65-mediated neurite outgrowth relates to the activation of FGFR1, p38 $8^{\mathrm{MAPK}}$ and ERK1/2. $\mathrm{Ca}^{2+}$, as an important second messenger, may also responsible for this process. Both the administration of ectoNP65 and enplastin increase the cytoplasmic $\mathrm{Ca}^{2+}$ concentration of cultured hippocampus neurons. Elevated $\left[\mathrm{Ca}^{2+}\right]_{\mathrm{i}}$ may further triggers the activation of $\mathrm{Ca}^{2+} /$ calmodulin-dependent protein kinase II (CaMKII) and supports the NP65-mediated neurite outgrowth. ${ }^{5}$

These finding indicate that FGFR signaling is required for NP65mediated neurite outgrowth. Nevertheless, whether NP65 can affect FGFR1 activity by directly binds to it remains elusive. Previous study has been confirmed that neuroplastin55, a neuroplastin isoforms lacks the first extracellular Ig set of NP65, binds to FGFR1 through its homologous sequence with FGFR1. Sequential similarities between those two molecules are in the first Ig set (Ig2) of neuroplastin55 and the first Ig set (Ig1) of FGFR $1 .{ }^{5}$ As mentioned above, NP65 shares similarities with FGFR1 and FGFR4. However, interplays between NP65 and FGFR still need more convincible evidences.

NP65 and synaptogenesis: In the nervous system, synaptic connections are indispensable for numerous neurons to form functional networks. The formation, maturation and maintains of synapse are regulated by signaling molecules both in the presynaptic and postsynaptic regions. Expression profiles of NP65 are in parallel to that of synaptophysin and PSD-95 during hippocampal development. ${ }^{12}$ Synaptic localizations of NP65 have been observed both in rodent and human brains, suggesting that it may involves in the development of synapse and the stabilization of synaptic contacts.

During the rat retinal development, it has been shown that NP65 is required for the synapse formation in the inner plexiform layer. ${ }^{4}$ In neuroplastin knockout mice, there was a decrease in the number of excitatory synapses both in the CA1 and DG regions of hippocampus. Increased mismatches between pre- and postsynaptic sites during synapse development were also detected in cultured hippocampal neurons derived from neuroplastin knockout mice. Moreover, the loss of synapse contacts might be mainly due to a decrease of postsynaptic apparatus rather than alterations in presynaptic terminals. NP65-Fc, a recombinant protein mimicked the ectodomain of the NP65, has been shown to interact with endogenous neurplastin 65 by homophilic binding to disturb Synaptogenesis and destabilize the established synapse contact in cultured hippocampal neurons, suggesting that interactions between NP65 could be affect by the treatment of exogenous NP65-Fc. Pre- and postsynaptic structures may not have influenced by another fusion protein-Np55-Fc, derived from neuroplastin55. ${ }^{7}$ Thus, NP65 might be the main forms of neuroplastin that are required for Synaptogenesis. Recently, studies have revealed that neuroplastin is also responsible for Synaptogenesis in inner hair cells of the mouse cochlea. The number of mismatched pre- and postsynaptic puncta was increased in $\mathrm{Nptn}^{\text {pitch/pitch }}$ mice, one type of mutant mice lacking the expression of NP65. ${ }^{8}$ In addition, reduced expression of PSD-95 was observed in NP65-deficient mice. In NP65deficient mice, the amount of synaptophysin seems unchanged. ${ }^{13}$ During the formation of synapses, recruitment of PSD-95 to the postsynaptic densities has been considered important for the initiation of postsynaptic differentiation. Further studies may be necessary to investigate whether interactions between NP65 and PSD-95 are responsible for the regulation of Synaptogenesis by NP65.

NP65 and synaptic plasticity: First evidence supporting roles of NP65 in synaptic plasticity came from the observation that maintenance of LTP in the hippocampal CA1 regions can be inhibited by treatment of NP65 antibodies and recombinant proteins derived from NP65, which may act as antagonists of pre-existing NP65 in the membrane to perturb their functions. Increased NP65 in the postsynaptic densities has also been observed after the induction of LTP. ${ }^{6}$ The mechanisms underlying these processes may relate to the activation of $\mathrm{p} 38^{\mathrm{MAPK}}$ and the internalization of AMPA receptors (Figure 1B). Treatment of NP65 fusion protein FcIg1-3 increases phosphorylation of p38 $8^{\mathrm{MAPK}}$ and inhibits LTP at hippocampal excitatory synapses. SB202190, a p $38^{\mathrm{MAPK}}$ inhibitor that blocks the activation of $\mathrm{p} 38^{\mathrm{MAPK}}$, can efficiently recue the formation and maintenance of LTP that inhibited by FcIg13. Meanwhile, application of FcIg1-3 also induces a reduction of surface AMPA receptor subunit GluR1, suggesting that internalization of AMPA receptors might be involved in FcIg1-3 induced prevention of LTP. ${ }^{14}$ The maintenance of hippocampal LTP is also disrupted in NP65-deficient mice. ${ }^{15}$ Together, these works established a role for NP65 in induction and maintenance of hippocampal LTP.

Figure 1 NP65 in neuronal maturation and synaptic plasticity. (A) Homophlic bindings between neuronplastin65 can induce neurite outgrowth during the maturation of neurons. Application of ectoNP65 or enplastin, which are derived from either the ectodomain or homophlic binding domains of NP65, have been shown to promote neurite outgrowth in vivo. Neruonplastin65 may also support Synaptogenesis, probably by interacting with other proteins in presynaptic sites or postsynaptic apparatus. (B) In hippocampal excitatory synapse, NP65 might interact with FGFR and induce activation of ERK1/2, CaMKII to promote LTP. Impaired LTP has been observed in NP65 deficient mice. Treatment of FcIg1-3 to disrupt functions of NP65 is also shown to inhibit LTP through activation of p38MAPK and the subsequent internalization of surface AMPA receptor. NP65, NP65; FGFR, fibroblast growth factor receptor; PSD95 , postsynaptic density protein-95; AMPAR, $\alpha$-amino-3-hydroxy5-methyl-4-isoxazole propionic acid (AMPA) receptor; NMDAR, $\mathrm{N}$-methyl-D-aspartate (NMDA) receptor; GABAR, $\gamma$-aminobutyric 
acid (GABA) receptor; CaMKII, $\mathrm{Ca}^{2+} /$ calmodulin-dependent protein kinase II; ERK1/2, extracellular signal-regulated kinas 1/2; LTP, longterm potentiation.

\section{Neuroplatin65 in maintenance of excitatory/inhibi- tory balance}

Excitatory/Inhibitory balances essential for the development and construction of neural circuits. ${ }^{16,17}$ Excitatory/Inhibitory (E/I) synaptic imbalance has been observed in mice lacking NP65.,13 Thus, neuropalstin 65 is involved in the regulation of $\mathrm{E} / \mathrm{I}$ balance.

NP65 might be contributing to the organization of excitatory synapses by regulating the trafficking and stabilization of glutamate receptors. Disruption of interactions between NP65 could trigger a reduction of surface GluR1 in hippocampal CA3 neurons, suggesting a role of NP65 in AMPA receptor trafficking. ${ }^{14}$ GluR1, together with
GluR2, is a predominant form of AMPA receptors at the hippocampal excitatory synapses. However, mechanisms underlying NP65 in the regulation of AMPA receptor trafficking have not been solved. NP65 is a putative mediator of NMDA receptor, which is another ionotropic glutamate receptor that widely distributed in the central nervous system. Compared to the significantly reduction of surface GluR1 subunits, application of FcIg1-3 only exhibited a less decrease in surface NR1 subunits of NMDA receptor. ${ }^{14}$ In contrast, recently studies indicated that there is an increase of NMDA receptor subunits including NR1, NR2A and NR2B in NP65-deficient mice. In NP65deficient mice, the expression level of NR2A subunit is relatively higher than that in wild-type mice. ${ }^{13}$ Meanwhile, hyper activation of ERK1/2 is also observed in NP65-decicent mice. Evidences have identified that NR2A can support ERK activation in mature neurons. ${ }^{18}$ Unfortunately, little is known about the precisely cellular mechanisms of these alterations.
A
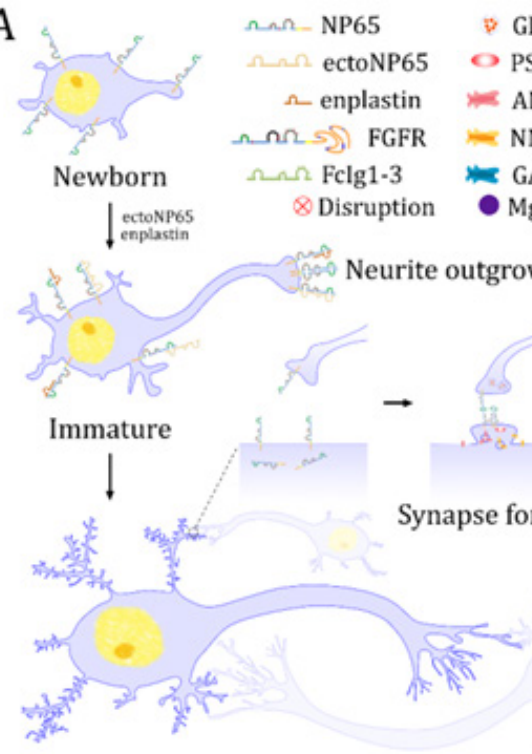

Mature

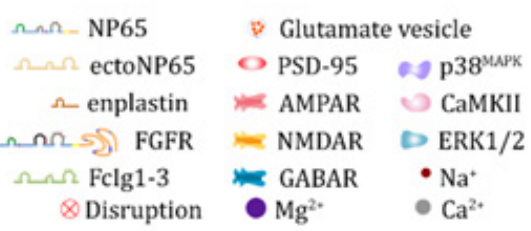

Neurite outgrowth

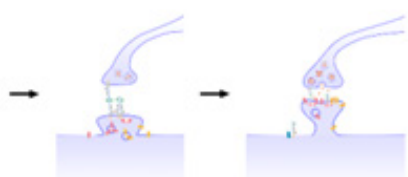

ynapse formation

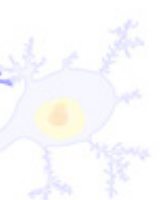

Figure I NP65 in neuronal maturation and synaptic plasticity.

At inhibitory synapses, NP65 is a potential organizer of $\gamma$-aminobutyric acid type $\mathrm{A}\left(\mathrm{GABA}_{\mathrm{A}}\right)$ receptors. Molecular interaction analyses by fluorescence resonance energy transfer (FRET) imaging revealed that NP65 is directly contact with $\mathrm{GABA}_{\mathrm{A}}$ receptor $\alpha 1, \beta 2$ and $\gamma 3$ subunits at cell surface of cultured HEK cells. In vitro studies also observed the co localization of NP65 with $\alpha 1, \alpha 2$ and $\alpha 5$ subunits in cultured hippocampal neurons. Interactions between NP65 and $\alpha 1$ subunit are localized at the synapse. Contact between NP65 and $\alpha 2$ subunit is mainly occurs at the synapse and sometime at the extra synaptic sites. Whereas, NP65 and $\alpha 5$ subunit have been shown to bind to each other in an extrasynaptic manner. In adult rats, NP65 is colocalized with $\beta 2$ and $\beta 3$ subunits in hippocampus. Application of NP65-shRNA to down regulated neruoplastin65 expressions produces a decrease in the number of $\alpha 2$ subunits at synapse. ${ }^{19}$ Mismatching between postsynaptic $\alpha 2$ subunits and presynaptic vesicular inhibitory amino acid transporter (VIAAT) is also increased in dendrites of neuroplastin-deficient mice. ${ }^{7}$ These observations support the idea that NP65 is involved in the regulation of GABA receptors probably by interact with their $\alpha 2$ subunits. GABA receptor $\alpha 2$ subunits are

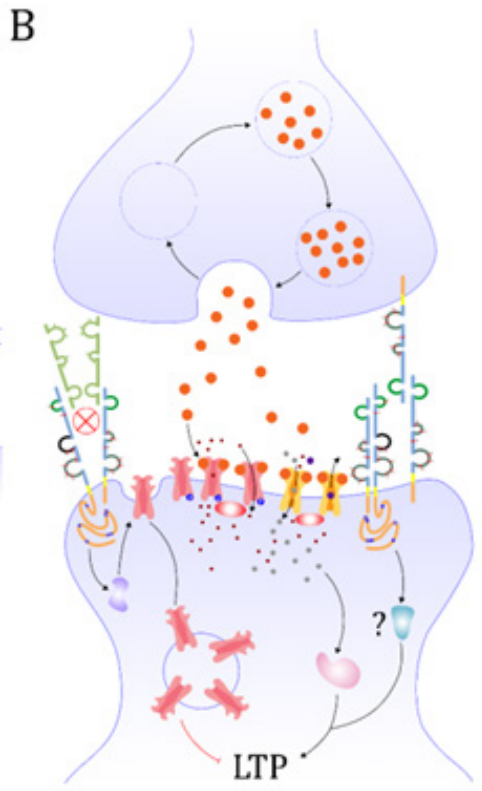

needed for the trafficking and accumulation of $\mathrm{GABA}_{\mathrm{A}}$ receptor clusters at inhibitory synapse. ${ }^{20,21}$ Thus, a deep understanding of interactions between NP65 and GABA receptors may reveal possible functions of NP65 in modulating development and dynamic changes of inhibitory synapses.

\section{NP65 and neuropsychiatric disorders}

NP65 is predominantly expressed in the cortex, hippocampus and amygdale. These brain regions are shown to governing mental processes including cognition and emotion. ${ }^{22}$ Functional roles of NP65 in development, synaptic plasticity and the maintenance of E/I balance also implying that dysfunction of NP65 could be possibly associate with some neuropsychiatric disorders.

\section{NP65 and cognitive disorders}

Cognitive disorders, such as dementia, are characterized by impairment of learning, memory, thinking, attention and other cognitive processes. Genetic studies have identified a single nucleotide polymorphism (rs7171755) located in the downstream of human 
NPTN gene is associated with cortical thickness in adolescents. It has been found that rs7171755 may affect NPTN expression. In adolescents, it has been found that cortex thickness and expression level of NPTN are higher in the right hemisphere compared with the left hemisphere. Variation at rs 7171755 in left hemisphere associates with lower cortical thickness and decreased intellectual abilities of adolescents. In the human prefrontal cortex, NPTN expression patterns are correlated with a variety of genes related to energy metabolism, synaptic transmission, learning and memory. ${ }^{11}$ NP65 is required for LTP at hippocampal synapse, suggesting its roles in learning and memory. First evidence provided by behavioral test demonstrates that enplastin, a peptide derived from NP65, may impair rat hippocampaldependent spatial learning at early phase by disrupting the functions of pre-existed NP65. ${ }^{14}$ Another study established that NP65 is required for the translocation of MCT2 to neuronal cell surface and support lactate transport. ${ }^{23}$ MCT2 is expressed both in the glia and the postsynaptic densities of synapse and provide energy supply for neurons. ${ }^{24,25}$

Emerging evidences support that alterations in brain energy metabolism may affect cognitive process. ${ }^{26,27}$ In rat model of Alzheimer's disease, decreased expression levels of MCT2 in the cerebral cortex and hippocampus was observed together with impaired learning and memory. ${ }^{28}$ Application of enplastin also induces the activation of $\mathrm{p} 38^{\mathrm{MAPK}}$, providing additional evidences that dysfunction of NP65 may relate to Alzheimer's disease. It has been reported that activation of $\mathrm{p} 38^{\mathrm{MAPK}}$ and caspase- 3 signaling probably involve in the onset of Alzheimer's disease. ${ }^{29}$ Our recent findings indicate that cognitive functions are altered in NP65-deficient mice. ${ }^{13}$ These studies highlighted that NP65 is closely related to several cognitive processes including intelligence, learning and memory. Whether dysfunctions of NP65 may associate with cognitive disorders, such as intellectual disability and Alzheimer's disease, is still need direct evidences.

\section{NP65 and anxiety}

Anxiety disorders are one of the most common mental disorders characterized by successively and elevated anxiety. Recent studies have revealed that NP65-deficient mice exhibit anxiety-like behaviors as validated by behavioral test, including the light/dark transition test, open field test and elevated plus maze test. Impaired fear memory is also observed in NP65-deficient mice..$^{13}$ Both the amygdala and the hippocampus are involved in acquisition and expression of fear conditioning, activity-dependent plasticity in these regions may contribute to the formation and consolidation of fear memories..$^{30-33}$

The hippocampus, the amygdala and the prefrontal cortex have been shown necessary for the extinction of learned fear. ${ }^{34,35}$ Thus, loss of NP65 in those brain regions would lead to alterations in fear memory and anxiety-related behaviors. In future studies, the challenge is to unravel the mechanisms of NP65 in regulating emotional processes like fear and anxiety.

\section{NP65 and schizophrenia}

Schizophrenia is a severe and highly heritable neuropsychiatric disorder with symptoms including delusions, hallucinations, abnormal thoughts, cognitive deficits and alteration in emotions. Genetic studies show that three SNPs (rs3840846, rs3826047 and rs3743500) located in the 5'-upstream regions of human NPTN gene may associate with schizophrenia. NPTN halotypes determined by those SNPs are differentially distributed in patient with schizophrenia. Halotype
T-G-T is under-represented in schizophrenia, whereas del-G-G is over-represented. Further in vitro studies confirm that halotype T-G-T has a lower transcriptional activity than del-G-G, suggesting that over expression of NPTN gene may relate to higher risks of schizophrenia. ${ }^{36}$ Impairment in pre pulse inhibition (PPI) of the auditory startle reflex is observed in neuroplastin-deficient mice, suggesting that loss of neuroplastin may contribute to schizophrenia-like phenotypes. ${ }^{37}$ Given its roles in regulation of neuronal development, synaptic contacts and maintenance of E/I balance, perhaps forthcoming discoveries could reveal that whether altered function of NP65 might account for schizophrenia.

\section{Conclusion}

Current researches focused on NP65 have significantly extended our knowledge of its structure, localization, interacting partners and the potential signal transduction pathways that are required for its functions. These findings acquired surprising evidences that NP65, as a member of Ig CAMs, is required for Neuritogenesis and Synaptogenesis during development. The known functions of NP65 are also related to the regulation of dendritic spine morphogenesis, synaptic plasticity and maintenance of E/I balance. However, our understanding of mechanisms underlying those processes is limited and some details need to be elucidated. For instance, NP65 is found to establish homophilic binding through its first extracellular Ig domain, but the binding domains of heterophilic interactions between NP65 with other proteins, like FGFR, GABA $A_{A}$ receptor and monocarboxylate transporter, is not determined yet. Furthermore, it is not clear whether there are putative binding domains in intracellular domains of neuroplatin 65 that are responsible for its functions. What intracellular molecules are involved in MAPK signaling pathway regulated by NP65 is another open question. It has been illustrated that NP65 may promote the activation of FGFRs and their downstream effector including ERK1/2, p $38^{\mathrm{MAPK}}$ and CREB. Nevertheless, ERK signaling and $\mathrm{p} 38^{\mathrm{MAPK}}$ signaling are two MAPK pathways that have distinct functions in the nervous system. Addressing this question might also shed light on our understanding of the roles of NP65 in regulation of synaptic plasticity. Recent advances have addressed that NP65 is important for hippocampal LTP probably by modulating neurotransmission and postsynaptic receptors trafficking. Insightful investigations could decipher the essential roles of neuoplastin65 in postsynaptic densities. In mice models, aberrant functions of NP65 may affect E/I balance and alterations in cognitive and emotional processes in mice models. Thus, sorting out the underlying mechanisms with further examination will possibly provide evidence of whether dysfunction of NP65 is related to neuropsychiatric disorders.

\section{Acknowledgements}

None.

\section{Conflict of interest}

The author declares no conflict of interest.

\section{References}

1. Chothia C, Jones EY. The molecular structure of cell adhesion molecules. Annu Rev Biochem. 1997;66:823-862.

2. Langnaese K, Beesley PW, Gundelfinger ED. Synaptic membrane glycoproteins gp65 and gp55 are new members of the immunoglobulin superfamily. J Biol Chem. 1997;272(2):821-827. 
3. Hill IE, Selkirk CP, Hawkes RB, et al. Characterization of nove glycoprotein components of synaptic membranes and postsynaptic densities, gp65 and gp55, with a monoclonal antibody. Brain Res. 1988;461(1):27-43.

4. Kreutz MR, Langnaese K, Dieterich DC, et al. Distribution of transcript and protein isoforms of the synaptic glycoprotein neuroplastin in rat retina. Invest Ophthalmol Vis Sci. 2001;42(8):1907-1914.

5. Owczarek S, Berezin V. Neuroplastin: cell adhesion molecule and signaling receptor. Int J Biochem Cell Biol. 2012;44(1):1-5.

6. Smalla KH, Matthies H, Langnase K, et al. The synaptic glycoprotein neuroplastin is involved in long-term potentiation at hippocampal CA1 synapses. Proc Natl Acad Sci U S A. 2000;97(8):4327-4332.

7. Herrera-Molina R, Sarto-Jackson I, Montenegro-Venegas C, et al Structure of excitatory synapses and GABAA receptor localization at inhibitory synapses are regulated by neuroplastin-65. J BiolChem. 2014;289(13):8973-8988.

8. Carrott L, Bowl MR, Aguilar C, et al. Absence of Neuroplastin-65 Affects Synaptogenesis in Mouse Inner Hair Cells and Causes Profound Hearing Loss. J Neurosci. 2016;36(1):222-234.

9. Bernstein HG, Smalla KH, Bogerts B, et al. The immuno localization of the synaptic glycoprotein neuroplastin differs substantially between the human and the rodent brain. Brain Res. 2007;1134(1):107-112.

10. Maness PF, Schachner M. Neural recognition molecules of the immunoglobulin super family: signaling transducers of axon guidance and neuronal migration. Nat Neurosci. 2007;10(1):19-26.

11. Desrivieres S, Lourdusamy A, Tao C, et al. Single nucleotide polymorphism in the neuroplastin locus associates with cortical thickness and intellectual ability in adolescents. Mol Psychiatry. 2015;20(2):263-274.

12. Buckby LE, Mummery R, Crompton MR, et al. Comparison of neuroplastin and synaptic marker protein expression in acute and cultured organotypic hippocampal slices from rat. Brain Res Dev Brain Res. 2004;150(1):1-7.

13. Amuti S, Tang Y, Wu S, et al. Neuroplastin 65 mediates cognitive functions via excitatory/inhibitory synapse imbalance and ERK signal pathway. Neurobiol Learn Mem. 2016;127:72-83.

14. Empson RM, Buckby LE, Kraus M, et al. The cell adhesion molecule neuroplastin-65 inhibits hippocampal long-term potentiation via a mitogen-activated protein kinase p38-dependent reduction in surface expression of GluR1-containing glutamate receptors. J Neurochem. 2006;99(3):850-860.

15. Wu S, Zhang H, Huang L, et al. Neuroplastin 65 knock out improves the cognitive function and inhibits LTP of mice. Int $J$ Dev Neurosci. $2015 ; 47: 8-9$

16. Dorrn AL, Yuan K, Barker AJ, et al. Developmental sensory experience balances cortical excitation and inhibition. Nature. 2010;465(7300):932936.

17. Keith D, El-Husseini A. Excitation Control: Balancing PSD-95 Function at the Synapse. Front MolNeurosci. 2008;1:1-4.

18. Kim MJ, Dunah AW, Wang YT, et al. Differential roles of NR2A- and NR2B-containing NMDA receptors in Ras-ERK signaling and AMPA receptor trafficking. Neuron. 2005;46(5):745-760.

19. Sarto-Jackson I, Milenkovic I, Smalla KH, et al. The cell adhesion molecule neuroplastin-65 is a novel interaction partner of gamma-aminobutyric acid type A receptors. J BiolChem. 2012;287(17):14201-14214.
20. Tretter V, Moss SJ. GABA(A) Receptor Dynamics and Constructing GABAergic Synapses. Front Mol Neurosci. 2008;1:1-7.

21. Jacob TC, Moss SJ, Jurd R. GABA(A) receptor trafficking and its role in the dynamic modulation of neuronal inhibition. Nat Rev Neurosci. 2008;9(5):331-343.

22. Pessoa L. On the relationship between emotion and cognition. Nat Rev Neurosci. 2008;9(2):148-158.

23. Wilson MC, Kraus M, Marzban H, et al. The neuroplastin adhesion molecules are accessory proteins that chaperone the monocarboxylate transporter MCT2 to the neuronal cell surface. PLoS One. 2013;8(11):e78654.

24. Gerhart DZ, Enerson BE, Zhdankina OY, et al. Expression of the monocarboxylate transporter MCT2 by rat brain glia. Glia. 1998;22(3):272281.

25. Chiry O, Fishbein WN, Merezhinskaya N, et al. Distribution of the monocarboxylate transporter MCT2 in human cerebral cortex: an immunohistochemical study. Brain Res. 2008;1226:61-69.

26. Cunnane S, Nugent S, Roy M, et al. Brain fuel metabolism, aging, and Alzheimer's disease. Nutrition. 2011;27(1):3-20.

27. Zlokovic BV. Neurovascular pathways to neurodegeneration in Alzheimer's disease and other disorders. Nat Rev Neurosci. 2011;12(12):723738.

28. Lu W, Huang J, Sun S, et al. Changes in lactate content and monocarboxylate transporter 2 expression in Abeta(2)(5)(-)(3)(5)-treated rat model of Alzheimer's disease. Neurol Sci. 2015;36(6):871-876.

29. Chen X, Lin R, Chang L, et al. Enhancement of long-term depression by soluble amyloid beta protein in rat hippocampus is mediated by metabotropic glutamate receptor and involves activation of p38MAPK STEP and caspase-3. Neuroscience. 2013;253:435-443.

30. Tsvetkov E, Carlezon WA, Benes FM, et al. Fear conditioning occludes LTP-induced presynaptic enhancement of synaptic transmission in the cortical pathway to the lateral amygdala. Neuron. 2002;34(2):289-300.

31. LeDoux J. The emotional brain, fear, and the amygdala. Cell MolNeurobiol. 2003;23(4-5):727-738.

32. Clugnet MC, LeDoux JE. Synaptic plasticity in fear conditioning circuits: induction of LTP in the lateral nucleus of the amygdala by stimulation of the medial geniculate body. J Neurosci. 1990;10(8):2818-2824.

33. Maren S, Quirk GJ. Neuronal signalling of fear memory. Nat Rev Neurosci. 2004;5(11):844-852.

34. Lesting J, Daldrup T, Narayanan V, et al. Directional theta coherence in prefrontal cortical to amygdalo-hippocampal pathways signals fear extinction. PLoS One. 2013;8(10):e77707.

35. Myers KM, Davis M. Mechanisms of fear extinction. Mol Psychiatry 2007;12(2):120-150.

36. Saito A, Fujikura-Ouchi Y, Kuramasu A, et al. Association study of putative promoter polymorphisms in the neuroplastin gene and schizophrenia. Neurosci Lett. 2007;411(3):168-173.

37. Bhattacharya S, Herrera-Molina R, Sabanov V, et al. Genetically Induced Retrograde Amnesia of Associative Memories After Neuroplastin Ablation. Biol Psychiatry. 2017;81(2):124-135. 